\title{
Electron microscopic and optical investigations of the indium distribution GaAs capped InxGa1-xAs islands
}

Woggon, U.; Langbein, Wolfgang Werner; Hvam, Jørn Märcher; Rosenauer, A.; Remmele, T.; Gerthsen, D.

Published in:

Applied Physics Letters

Link to article, DOI:

10.1063/1.119542

Publication date:

1997

Document Version

Publisher's PDF, also known as Version of record

Link back to DTU Orbit

Citation (APA):

Woggon, U., Langbein, W. W., Hvam, J. M., Rosenauer, A., Remmele, T., \& Gerthsen, D. (1997). Electron microscopic and optical investigations of the indium distribution GaAs capped InxGa1-xAs islands. Applied Physics Letters, 71(3), 377-379. https://doi.org/10.1063/1.119542

\section{General rights}

Copyright and moral rights for the publications made accessible in the public portal are retained by the authors and/or other copyright owners and it is a condition of accessing publications that users recognise and abide by the legal requirements associated with these rights.

- Users may download and print one copy of any publication from the public portal for the purpose of private study or research.

- You may not further distribute the material or use it for any profit-making activity or commercial gain

- You may freely distribute the URL identifying the publication in the public portal 


\title{
Electron microscopic and optical investigations of the indium distribution in GaAs capped $\ln _{x} \mathrm{Ga}_{1-x}$ As islands
}

\author{
U. Woggon ${ }^{\text {a) }}$ \\ Institut für Angewandte Physik der Universität Karlsruhe, 76128 Karlsruhe, Germany \\ W. Langbein and J. M. Hvam \\ Mikroelektronik Centret, DTU, DK-2800 Lyngby, Denmark
}

\author{
A. Rosenauer, T. Remmele, and D. Gerthsen \\ Laboratorium für Elektronenmikroskopie, Universität Karlsruhe, 76128 Karlsruhe, Germany
}

(Received 2 December 1996; accepted for publication 19 May 1997)

\begin{abstract}
Results from a structural and optical analysis of buried $\operatorname{In}_{x} \mathrm{Ga}_{1-x}$ As islands carried out after the process of GaAs overgrowth are presented. It is found that during the growth process, the indium concentration profile changes and the thickness of the wetting layer emanating from a StranskiKrastanow growth mode grows significantly. Quantum dots are formed due to strong gradients in the indium concentration, which is demonstrated by photoluminescence and excitation spectroscopy of the buried $\operatorname{In}_{x} \mathrm{Ga}_{1-x}$ As islands. (C) 1997 American Institute of Physics.
\end{abstract}

[S0003-6951(97)03029-5]

The spontaneous formation of small islands during the epitaxial growth on lattice-mismatched substrates is at present a subject of intensive research. ${ }^{1-3}$ The exact determination of the indium concentration in the grown structure, however, is a problem, particularly after the final overgrowth of the islands with the GaAs cap layer. ${ }^{4}$ In this letter we present a structural analysis of buried $\operatorname{In}_{x} \mathrm{Ga}_{1-x}$ As islands by cross-sectional high-resolution transmission electron microscopy (HRTEM) after overgrowth with a GaAs cap layer. Both the dimension and shape as well as the indium concentration profile have been determined. We show that the overgrown structure can be considered as $\operatorname{In}_{x} \mathrm{Ga}_{1-x} \mathrm{As}$ islands with higher In-content than the surrounding $\operatorname{In}_{y} \mathrm{Ga}_{1-y} \mathrm{As}$ quantum well $(\mathrm{QW})(x>y)$. The original wetting layer grows to about 10 monolayers (MLs) thick QW and encloses the islands. The optical properties clearly show that the islands maintain their quantum dotlike behavior after the capping with a thin GaAs layer. The surrounding QW acts as a carrier transport layer for the excitation of the buried quantum dots with the higher In content. Photoluminescence excitation spectra show the longitudinal optical (LO) phononassisted relaxation process in the energy states of the $\mathrm{In}_{x} \mathrm{Ga}_{1-x}$ As islands.

The investigated sample has been grown in a standard growth process by molecular beam epitaxy on a GaAs (001) substrate at $T_{\text {subst }}=500{ }^{\circ} \mathrm{C}$ with a $200 \mathrm{~nm}$ thick GaAs buffer layer. The nominal composition and layer thickness have been chosen to be $x=0.6$ and $1.5 \mathrm{~nm}$, respectively. For that growth procedure, the occurrence of the three-dimensional (3D) Stranski-Krastanow growth mode has been proven in a previous analysis ${ }^{5}$ carried out at samples without a GaAs cap layer. These samples, however, are optically inactive due to surface recombination. To have a direct comparison between optical and structural properties, an optically active, overgrown sample is analyzed in a transmission electron microscopy (TEM) (Philips CM20 FEG electron microscope operated at $200 \mathrm{kV}$ acceleration voltage). Figure 1(a) shows a [004] dark-field image of the $\operatorname{In}_{x} \mathrm{Ga}_{1-x}$ As layer and the

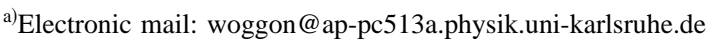

GaAs cap layer. The dark spots that are marked by black arrows are due to strain fields inside the substrate. The average distance is about $15 \mathrm{~nm}$. Islands towering above the wetting layer are not visible. Furthermore, the capping layer shows a very smooth surface which can also be seen in the HRTEM image in Fig. 1(b). The image does not provide any chemical contrast. In order to obtain quantitative information concerning layer thicknesses and In concentration $x$, digital analysis methods have been applied. The procedure used is a combination of the methods DALI, ${ }^{6}$ QUANTITEM $^{7}$ and the procedure proposed in Ref. 8 and is described in detail in Ref. 9. First, the positions of the intensity maxima of the bright spots in Fig. 1(b) are calculated. The obtained lattice site positions are correlated with the positions of the atomic columns. A regular, equally spaced reference lattice formed by the $\{220\}$ and (002) planes is calculated from a reference region inside the substrate and then expanded over the whole image. The displacement vector of each lattice site results from the evaluation of the distance vectors between the experimental lattice site and the reference lattice site belonging to it. Figure 1(c) is a color coded map of the resulting [001] components of displacement vectors. The displacements vanish inside the (blue) region of the substrate. Since the lattice parameter of $\operatorname{In}_{x} \mathrm{Ga}_{1-x}$ As is larger than that of GaAs, an increasing displacement is found inside the wetting layer (light blue to green). The red spot emanates from a region of increased In content inside the wetting layer that gives rise to a lattice bending in the [001] direction inside the cap layer. Figure 1(d) displays a color coded map of local lattice parameters that are calculated from lattice site distances in the [001] direction and clearly shows the broadened wetting layer (light blue to green) which contains a (red) region of increased lattice parameter corresponding to the region below the red spot in Fig. 1(c). Figure 2 shows the displacements averaged along the $\langle 110\rangle$ direction for each monolayer between the top (number 1) and the bottom (number 75) of regions $\mathrm{A}$ and $\mathrm{B}$. As mentioned above, the regions of increasing displacements belong to the $\mathrm{In}_{x} \mathrm{Ga}_{1-x}$ As region and its gradient is a measure for the In content $x$ that is clearly increased for region B (triangles in Fig. 2). However, the thickness of these regions is about 10 MLs for both A and B. 

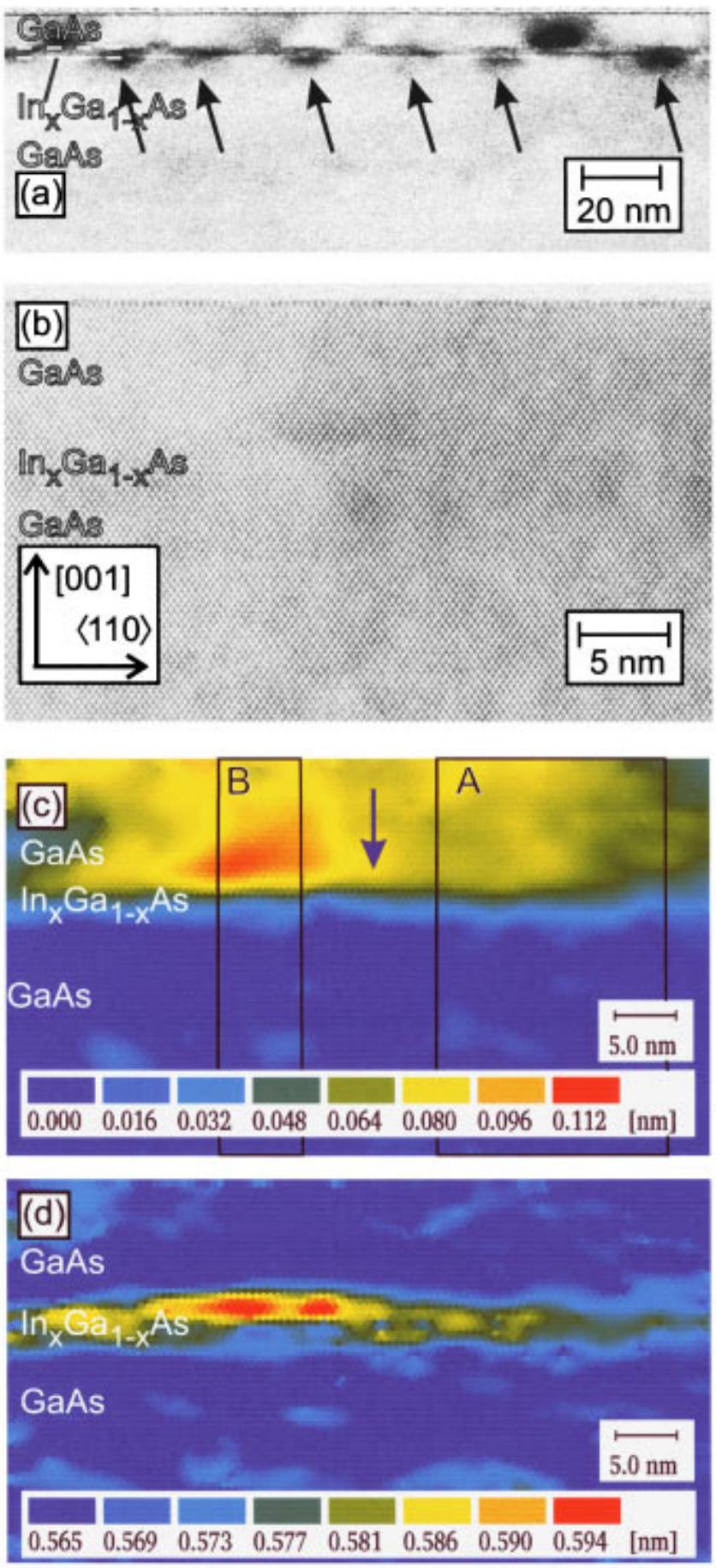

FIG. 1. (a) TEM [004] dark-field image showing the $\mathrm{In}_{x} \mathrm{Ga}_{1-x}$ As layer and the $10 \mathrm{~nm}$ thick GaAs cap layer. The black arrows mark dark spots that are due to strain fields inside the substrate. (b) $\langle 110\rangle$ HRTEM image that contains the GaAs substrate, the $\operatorname{In}_{x} \mathrm{Ga}_{1-x}$ As layer and the GaAs cap layer. (c) Color coded map of [001] components of local displacements. The displacements are obtained by a DALI evaluation of the HRTEM image shown in (b). Regions A and B correspond to solid circles and open triangles in Fig. 2, respectively. The blue arrow indicates the direction of increasing (002) plane numbers in Fig. 2. Region B contains an area of increased In content. (d) Color coded map of local lattice parameters. The maximum lattice parameter of $0.594 \mathrm{~nm}$ corresponds to a strain of $\epsilon_{\perp}=0.051$.

Because a direct analytical calculation of the In content is not possible due to the tetragonal distortion of the strained layer and its elastic relaxation, a finite element (FE) calculation had to be performed which will be described in detail elsewhere. The averaged sample thickness of $10 \mathrm{~nm}$ in electron beam direction was evaluated from Fig. 1(b) with the

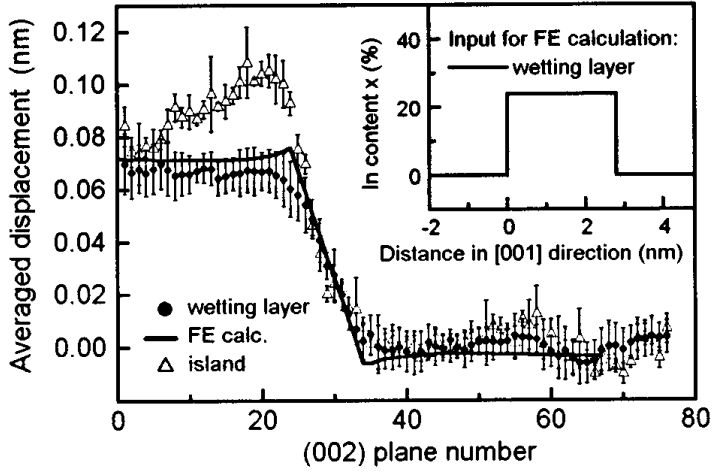

FIG. 2. The solid circles represent the [001] components of local displacements averaged along the $\langle 110\rangle$ direction inside region A in Fig. 1, plotted versus the (002) plane number. The solid line is the result of the FE calculations which have been carried out with an In concentration profile as shown in the insert. The open triangles show the displacements averaged inside region $\mathrm{B}$.

procedure proposed in Ref. 9 and used for the FE calculations. A best fit to the displacements averaged in region A (circles in Fig. 2) could be obtained for the In concentration profile as shown in the insert of Fig. 2 with a constant In content of $y=0.24$. For the maximum In content of the (inhomogeneous) buried island (region B) the value $x=0.6$ is obtained.

In the following, the optical properties of this structure are studied by photoluminescence (PL) and PL excitation (PLE) spectroscopy. The energy of the optical transitions of the $\mathrm{In}_{y} \mathrm{Ga}_{1-y}$ As quantum structures is influenced both by their thickness and In composition $y$. This is quantified in the inset of Fig. 3, which shows the calculated energies for the transition between the lowest confined electron and hole states in coherently strained $\operatorname{In}_{y} \mathrm{Ga}_{1-y} \mathrm{As} / \mathrm{GaAs}$ quantum wells. In the calculation we employed an isotropic effective mass model for the conduction band and a 6-band $\mathbf{k} \cdot \mathbf{p}$ model for the valence band, using the strain matrix elements and parameter given in Refs. 1 and 10. As can be seen in Fig. 3, the calculation predicts a transition energy of $1.4 \mathrm{eV}$ for the $\mathrm{In}_{y} \mathrm{Ga}_{1-y} \mathrm{As} \mathrm{QW}$ which surrounds the islands $(y=0.24$, $d=2.8 \mathrm{~nm}$, see Fig. 2). The buried $\operatorname{In}_{x} \mathrm{Ga}_{1-x}$ As islands exhibit a higher indium concentration, and should consequently have transition energies below $1.4 \mathrm{eV}$. The experimental transition energies are determined by the low-temperature PL and PLE spectra shown in Fig. 3. The PL has been excited above the GaAs band gap and shows a broad band with the maximum at $1.2 \mathrm{eV}$ (dashed line). This PL we attribute to the $\mathrm{In}_{x} \mathrm{Ga}_{1-x} \mathrm{As}$ islands, which efficiently capture the electronhole pairs excited in the surrounding QW and the GaAs cap layer. The low-temperature PL spectrum shows no signal from the surrounding QW, revealing the fast carrier relaxation into the islands. To decrease the influence of inhomogeneous broadening caused by variation in island geometry and In concentration, energy-selective PLE has been applied by tuning the detection energy within the PL band. Two characteristic types of peaks are observed in the PLE spectra of Fig. 3: One is spectrally constant at $1.4 \mathrm{eV}$ and corresponds to the calculated energy of the surrounding $2.8 \mathrm{~nm}$ thick $\operatorname{In}_{0.24} \mathrm{Ga}_{0.76} \mathrm{As} \mathrm{QW}$. It is due to the absorption into this QW followed by relaxation into the islands. The other features follow the detection energy and are thus correlated with 


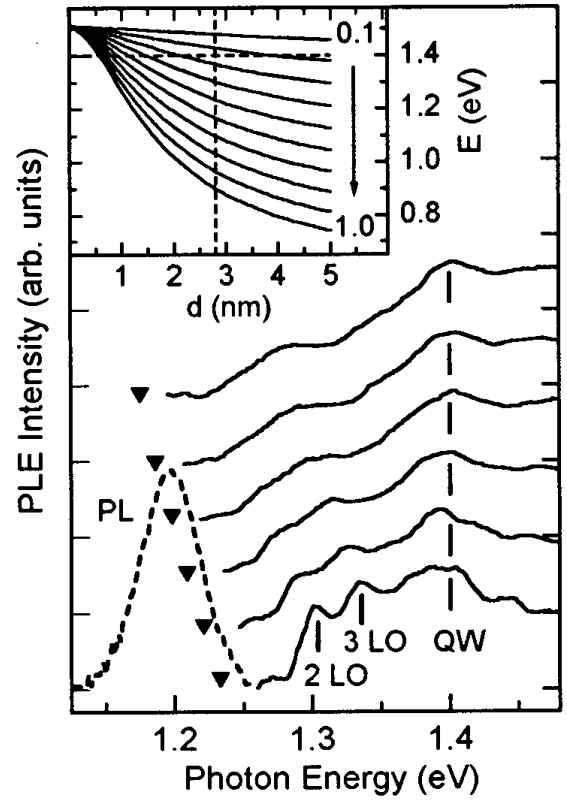

FIG. 3. PLE spectra (solid lines) recorded at different energy positions within the inhomogeneously broadened PL band (dashed line) of the buried $\mathrm{In}_{x} \mathrm{Ga}_{1-x} \mathrm{As}$ islands measured at $T=5 \mathrm{~K}$. The inset shows the calculated lowest transition energies $\mathrm{E}$ of strained $\mathrm{In}_{y} \mathrm{Ga}_{1-y} \mathrm{As} / \mathrm{GaAs}$ quantum wells as a function of well thickness $d$ with the indium concentration $y$ as the parameter varied in steps of 0.1 between 0.1 and 1 .

electronic states of the selectively detected buried islands. The PLE signal below the QW energy arises from the coexistence of absorption in excited states of the islands and phonon-assisted absorption and relaxation processes. The excited state energies have been calculated assuming circular islands and a step potential barrier giving an energy separation between ground and first excited state between 30 and $50 \mathrm{meV}$. This uncertainty in energy is the result of fluctuations in composition, shape and size of the islands, even for a fixed ground state energy. The energy distribution becomes even broader for the second excited state which is expected to be $\sim 100 \mathrm{meV}$ above the ground state. Thus, the broad maxima visible in PLE when detecting below the PL maximum we explain by absorption into excited states having a broad energy distribution. For PLE detection above the PL maximum energy $\left(\mathrm{E}_{\mathrm{DET}}^{\mathrm{PLE}}=1.23 \mathrm{eV}\right)$, three distinct peaks at $\mathrm{E}=1.270,1.302$, and $1.335 \mathrm{eV}$, are visible. We attribute them to a phonon-assisted absorption and relaxation process involving both InAs- and GaAs-type phonons. The stronger contribution of the LO phonon relaxation at higher detection energies is attributed to a stronger relaxation to lower lying electronic states reducing the influence of the slower multiphonon relaxation on the population of the monitored states.

Finally, we discuss the consequences of the overgrowth process for the quantum dot character of the buried islands. Before the deposition of the cap layer, the grown 10-20 nm wide islands can clearly be recognized in HRTEM. ${ }^{5}$ Do these quantum structures maintain their zero dimensionality or does the final overgrowth process result in their dissolution? To answer this question we investigate in the following the density of states (DOS), which can be used as a fingerprint for the dimensionality of the grown structures. Zero dimensionality or "quantum dot" behavior can be stated if we find a drastic reduction in the DOS due to the small active vol-

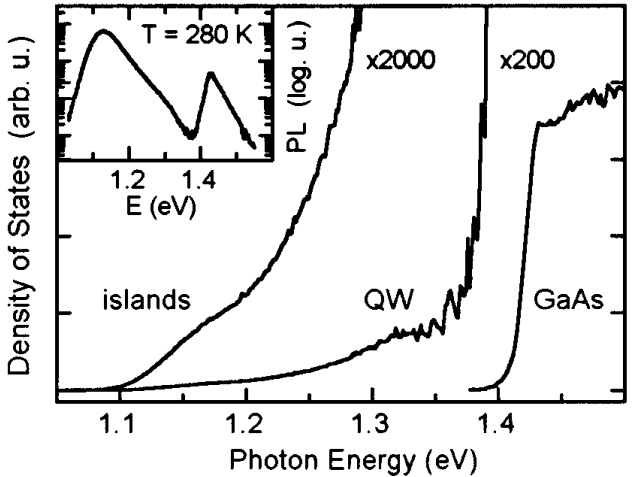

FIG. 4. Combined DOS derived from the PL spectrum at $T=280 \mathrm{~K}$ (inset), $\lambda_{\mathrm{exc}}=488 \mathrm{~nm}, I_{\mathrm{exc}}=3 \mathrm{~kW} / \mathrm{cm}^{2}$.

ume and a $\delta$-like behavior. To obtain information about the DOS we analyze the PL at a high lattice temperature and assume a nondegenerate thermal population of all available states. In this manner we can estimate the combined DOS by the relation $\mathrm{DOS} \propto I_{\mathrm{PL}}(\hbar \omega) \exp \left(\hbar \omega / k_{B} T\right)$ assuming a constant optical transition strength. The result is plotted in Fig. 4. A sharp drop to lower energies at the GaAs band edge is due to the reduction of the effective volume from the bulk substrate with a 3D DOS to the wetting layer QW with a 2D DOS. Below the energy of the QW, the DOS does not exhibit a featureless tail as expected for random disorder within the QW due to composition and well width inhomogeneities. In contrast, we detect a lower lying structure at the transition energy of the buried islands. Its combined DOS is about one order of magnitude less than that of the QW, which indicates a high density of quantum dots corresponding to a filling factor of about $10 \%$, in agreement with the HRTEM results (Fig. 1). Thus, the overgrowth process changes the confinement conditions but does not destroy the quantum dots. For the analysis of the transition energies, however, knowledge of the actual indium concentration within the barrier and the island is crucial, in particular if the process of indium diffusion is likely during the overgrowth.

This work has been supported by the Deutsche Forschungsgemeinschaft, the Volkswagenstiftung (Contract No. I/71014) and by the Danish Ministries of Research and Industry in the framework of CNAST. We are grateful to A. Förster for growing the samples.

${ }^{1}$ M. Grundmann, O. Stier, and D. Bimberg, Phys. Rev. B 52, 11969 (1995).

${ }^{2}$ S. Fafard, D. Leonard, J. L. Merz, and P. M. Petroff, Appl. Phys. Lett. 65, 1388 (1994)

${ }^{3}$ R. Nötzel, J. Temmyo, A. Kozen, T. Tamamura, T. Fukui, and H. Hasegawa, Adv. Solid State Phys. 35, 103 (1995).

${ }^{4}$ R. Leon, Y. Kim, C. Jagadish, M. Gal, J. Zhou, and D. J. H. Cockayne, Appl. Phys. Lett. 69, 1888 (1996).

${ }^{5}$ K. Tillmann, D. Gerthsen, P. Pfundstein, A. Förster, and K. Urban, J. Appl. Phys. 78, 3824 (1995).

${ }^{6}$ A. Rosenauer, S. Kaiser, T. Reisinger, J. Zweck, and W. Gebhardt, Optik (Stuttgart) 102, 63 (1996).

${ }^{7}$ C. Kisielowski, P. Schwander, F. H. Baumann, M. Seibt, Y. Kim, and A. Ourmazd, Ultramicroscopy 58, 131 (1995).

${ }^{8}$ D. Stenkamp and H. P. Strunk, Appl. Phys. A 62, 369 (1996).

${ }^{9}$ A. Rosenauer, T. Remmele, D. Gerthsen, K. Tillmann, and A. Förster, Optik 105, 99 (1997).

${ }^{10}$ M.-E. Pistol, M. Gerling, D. Hessman, and L. Samuelson, Phys. Rev. B 45, 3628 (1992).

${ }^{11}$ R. Heitz, M. Grundmann, N. N. Ledentsov, L. Eckey, M. Veit, D. Bimberg, V. M. Ustinov, A. Yu. Egorov, A. E. Zhukov, P. S. Kopev, and Zh. I. Alferov, Appl. Phys. Lett. 68, 361 (1996). 